\title{
Research of the Spectral Characteristics of Apodized Fiber Bragg Gratings
}

\author{
Maksat Kalimoldayev ${ }^{1}$, Aliya Kalizhanova ${ }^{1,3}$, Waldemar Wójcik $^{1,2}$, Gulzhan Kashaganova ${ }^{1,4}$, Saltanat Amirgaliyeva ${ }^{1}$, \\ Azhibek Dasibekov ${ }^{5}$, Ainur Kozbakova ${ }^{1,3}$, Zhalau Aitkulov ${ }^{1}$ \\ ${ }^{1}$ Institute of Information and Computational Technologies CS MES RK, Kazakhstan, \\ ${ }^{2}$ Lublin Technical University, Poland \\ ${ }^{3}$ Al-Farabi Kazakh National University, Kazakhstan \\ ${ }^{4}$ Kazakh-American University, Kazakhstan \\ ${ }^{5}$ M.Auezov South Kazakhstan State University
}

\begin{abstract}
Fiber Bragg Gratings (FBG) are widely used in different areas of the state-of-the -art fiber optics. Every task imposes specified requirements to the FBG spectral characteristics, which are scheduled at the gratings manufacturing stage. Manufacturing and using the Bragg fiber-optic gratings is impossible without measuring their characteristics at every stage of manufacturing the gratings themselves and devices on their basis. To select FBG's optimal parameter we will compare the parameter SGW with several different the most widely used apodization functions. Upon manufacturing the FBG there applied strict requirements to their parameters. Recording or manufacturing the fiber Bragg gratings might be classified according to the type of the laser being used, radiation wave length, recording techniques, irradiation material and grating type. The article is dedicated to the techniques of computing and measuring the FBG's principal parameters; it is necessary to define optimal parameters of the characteristics for the grating quality operation.
\end{abstract}

\section{Introduction}

Let's choose the apodized FBG as an example. Bragg's apodized gratings. Bragg's homogeneous gratings spectrum, being an addition to the base peak, also has undesirable high indirect side lobes, which generate cross-interference between neighboring channels.

FBG definite applications impose certain requirements to the reflectance spectrum form, which is specified by the grating refractive index profile, one of the common requirements to them is absence of side lobes. The phenomenon thereof is undesirable, because it strives to the wave liquidation.

Appearance of side lobes is connected with the fact that the Bragg grating structure, which has a finite length, quickly starts and finishes. It is of paramount importance to eliminate those side lobes reflective factors and bring it to the minimum. The most widely used method to eliminate the side lobes is the Bragg fiber gratings apodization. It means, that the reflection coefficient amplitude is modulated in such a manner, that it increases and decreases gradually in compliance with the applied function. Thus, the apodization is a highly valued instrument for smoothing the Bragg grating reflection spectrum, but it also influences at the gratings dispersion characteristics. Apodization might be reached by means of UV light action.

As it has been described above, an important element, defining the Bragg gratings quality is the size of the so-called side lobes, occurring in their spectral characteristics. In order to specify the gratings' quality let's introduce a new parameter which is called SGW.

\section{Bragg's apodized gratings}

To select FBG's optimal parameter we will compare the parameter $\mathrm{S}_{\mathrm{GW}}$ with several different the most widely used apodization functions. Upon manufacturing the FBG there applied strict requirements to their parameters. Paramount parameters of the fiber gratings is distribution of $\Delta n(\mathrm{x})$ reflection factor modulation amplitude and the law of the grating change period $\Delta \Lambda(\mathrm{x})$ along the fiber longitudinal axis. Those parameters deviation from the demanded values brings to worsening the FBG spectral characteristics.

Recording or manufacturing the Bragg fiber gratings might be classified according to the type of the laser being used, radiation wave length, recording techniques, irradiation material and grating type [1].

Lasers, used for FBG recording might be both uninterrupted and impulse with radiation wave length from intra-red (IR) to ultraviolet (UV) spectrum range. Difference data specifies spatial and time coherence of optical radiation sources being used for recording, which in its turn defines the selection of FBG recording appropriate method. Among the base methods of FBG recording there is pointed out the phase mask method $(\mathrm{PhM})$, interferometer method and step-by-step approach.

\footnotetext{
Corresponding author: ainur79@mail.ru
} 
Phase mask method is the simplest and most effective as it allows excluding from the recording scheme the expensive vibro-isolating tables, vibroisolated foundation and footings, necessary upon multipulsed recording, and at that, receiving gratings with required characteristics (Fig. 1). At the first stage the phase mask is formed by means of flashing the quartz plate covered with photoresist material. Flashing is fulfilled either with an electronic beam, controlled by a computer, or by forming interference image mask on the photoresist material surface. Only after the flashed plate etching there is formed the mask with necessary period $\Lambda_{\mathrm{M}}$.

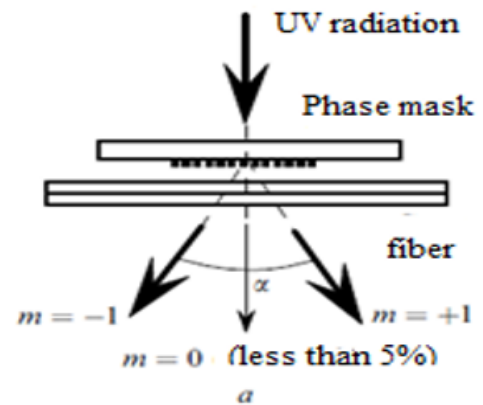

Fig. 1. Scheme of FBG recording by means of phase mask (direct recording).

At the second stage there is formed the diffraction grating in the fiber [2], [3]. For that purpose the ultraviolet beam from the irradiation source is directed on the phase mask surface, light diffraction, on which there applied a number of light beams of different orders.

In virtue of FBG short period they are manufactured, as a rule, using interference methods (Fig. 2). As the necessary grating structure recording process might last a few tens of minutes, the quality grating manufacturing is possible at interference picture high stability.Use a two-column format, and set the spacing between the columns at $8 \mathrm{~mm}$. Do not add any page numbers.

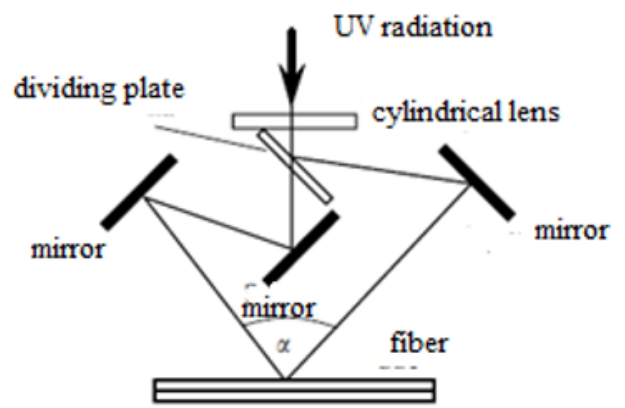

Fig. 2. Scheme of FBG recording in interferometer with beam amplitude separation of UV radiation.

Such conditions might be secured only under appropriate spatial and temporal coherence of photo-inducing radiation, which anticipates strict requirements to radiation sources for FBG recording.

One more recording method is the step-by-step approach. This method's attractiveness in that it eliminates the necessity of using the phase mask and allows recording the Bragg gratings with a resonance at any wave length [4].

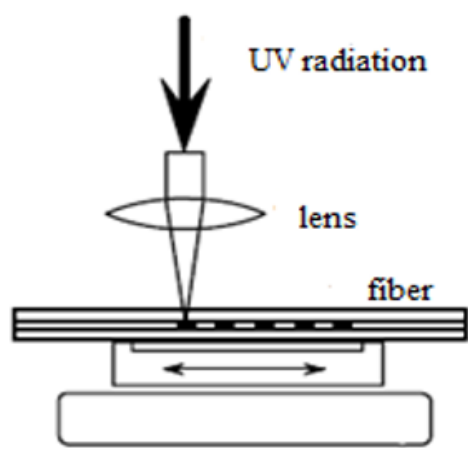

Fig. 3. Scheme of FBG recording by means of step-by-step approach.

Apart from that, the method thereof allows (Figure 3) forming any profiles of grating's separate slit and total distribution of the amplitude of directed Refraction Index (RI) in whole, as well changing the period along the grating length [5], i.e., creating the chip FBG without using phase mask with the period variable along the length.

Growing interest to the Bragg optic fiber gratings results in occurrence of their multiple varieties.

The most important property of the Bragg fiber gratings is the narrow-band optical radiation, the relative spectral width of which might constitute $10^{-6}$ and less. Fiber gratings advantages comparing to the alternative technologies (for instance, interference mirrors and volume gratings) are obvious: the wide variety of being received spectral and dispersive characteristics, most of which can be implemented only based on the fiber gratings refraction factor; completely fiber fulfillment; low optical losses; relative ease of fabrication and others.

The Bragg's ideal fiber grating functioning is passing the beam crosswise, excluding passing the wave's one length infinitely thin. Only the wave's lengths, satisfying Bragg's condition will be reflected and spread out in opposite direction. Sure enough, transmission and characteristics of reflection are distorted by the so called side lobes. This phenomenon, as it is shown in the Figure 4 , is undesirable, as it strives to the wave liquidation.

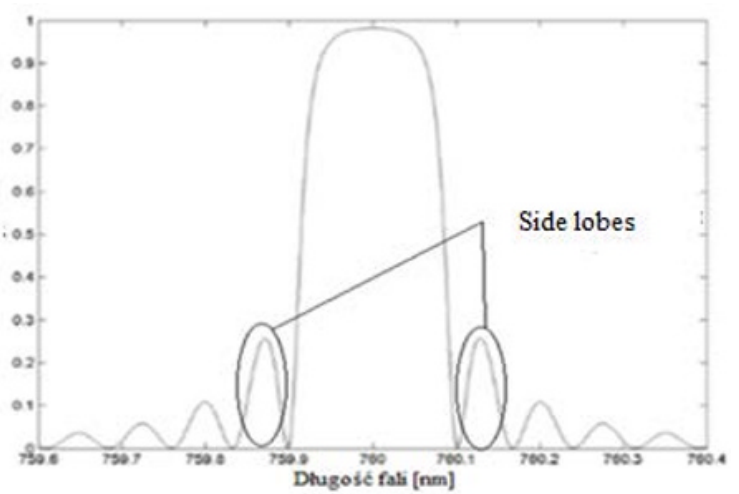

Fig. 4. Reflection spectrum of Bragg fiber grating

It is very important to eliminate reflecting power of the side lobes thereof and lead them to the minimum. 
The most widely used method for eliminating the side lobes is Bragg fiber grating apodization [8]. It means, that refraction index amplitude is modulated in such a manner, that it grows and decreases in compliance with the applied function [11]. Thus, apodization is a valuable tool for smoothing the Bragg grating reflection spectrum but, as well, influences at grating dispersive characteristics. Apodization can be reached by means of UV-radiation impact.

Fig. 5 shows the difference in sizes of the side lobes of Bragg fiber gratings reflection spectrum with apodization and without it.
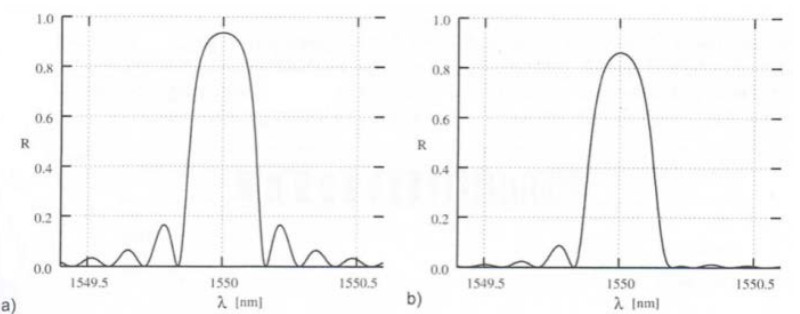

Fig. 5. Reflection spectrum of Bragg fiber grating a) without apodization, b) apodized

In case of apodized Bragg gratings the amplitude modulation increases and gradually decreases. Under amplitude modulation method the ups and downs speed occurs along the fiber axis and depends on the apodization function.

The technology thereof has opened new possibilities for creating and optimizing the fiber-optic elements, in particular, the filters, dispersion compensation modules, lasers with the distributed feedback and fiber-optic sensors for different parameters measuring.

The most widely used method for eliminating the side lobes is the Bragg fiber gratings apodization.

Side lobes suppression is reached at the expense of securing the smooth modulation amplitude change and leveling the average value of the directed refraction index along the grating, the so called grating profile apodization.

There exist a lot of refraction index profiles, which allow receiving the FBG spectrum with suppressed side lobes [9], at that, practical implementation of most of them requires technologically complicated scanning techniques.

Apodization primary functions or apodization profiles and their formulae:

- Gauss function $g(z)=\exp \left[-a\left(\frac{z-\frac{L}{2}}{L}\right)^{2}\right]$;

- Raised sine function $g(z)=\sin ^{2}\left(\frac{\pi z}{L}\right)$;

- Sinc function $g(z)=\operatorname{sinc}^{X}\left(\left|\frac{2(z-L / 2)}{L}\right|^{Y}\right)$;

- Tanh function $g(z)=1+\tanh \left[T\left(1-2\left(\frac{z}{L_{g}}\right)^{\alpha}\right)\right]$;
- Blackman function $g(z)=0,42+0,5 \cos \left(\frac{\pi z}{a}\right)+$ $\frac{2}{25} \cos \left(\frac{2 \pi z}{a}\right)$;

- Hamming function $g(z)=\frac{1+H \cos \left(\frac{2 \lambda z}{l}\right)}{1+H}$;

- Cosine function $g(z)=\cos ^{A}\left(\frac{\pi}{L_{g}} z\right)$;

- Cauchy function $T(z)=\frac{1-\left(\frac{2 z}{L_{g}}\right)^{2}}{1-\left(\frac{2 B z}{L_{g}}\right)^{2}}$.

The most widely applied apodization functions are: Gauss function, Sinc function, Blackman function and Cosine function.

Let's consider the widely used apodization functions and their influence at spectral characteristics of Bragg fiber gratings.

Gauss function is expressed by the formula [9]:

$$
g(z)=\exp \left[-a\left(\frac{z-\frac{L}{2}}{L}\right)^{2}\right]
$$

\subsection{Numerical solution}

Examples of Gauss profiles for various parameters values $a$ are represented in Fig. 6, 7, 8, 9.

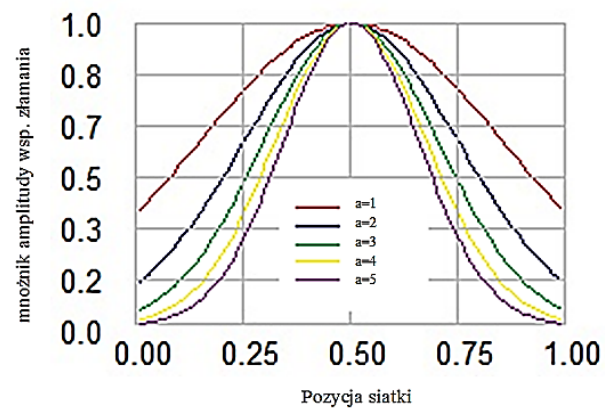

Fig. 6. Gauss apodization profiles for different values of parameter $a$.

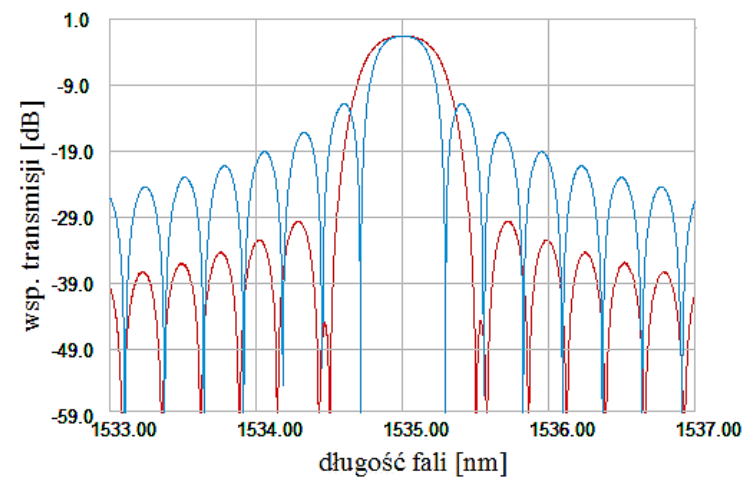

Fig. 7. Comparison of Bragg fiber gratings characteristics without apodization - blue line and apodized Gauss function at $\mathrm{a}=2$ - red line. 


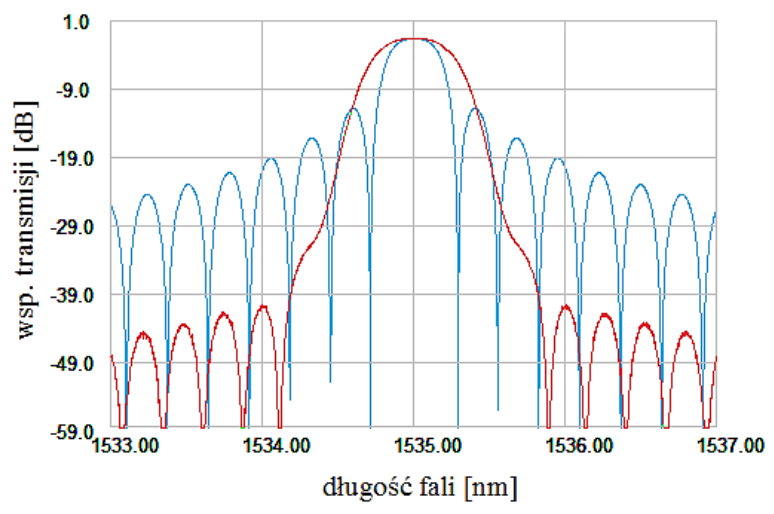

Fig. 8. Comparison of Bragg fiber gratings spectral characteristics without apodization - blue line and apodized Gauss function at $\mathrm{a}=3$ - red line.

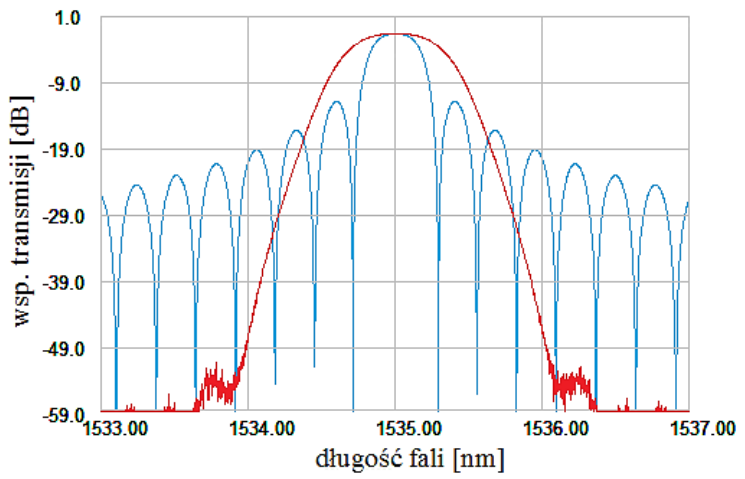

Fig. 9. Comparison of Bragg fiber gratings spectral characteristics without apodization - blue line and apodized Gauss function at $\mathrm{a}=5$ - red line.

Figures 7, 8 and 9 show differences in Bragg fiber gratings spectral characteristics without apodization and with apodization using Gauss function with different values of the parameter a. From the schemes thereof it is seen, that the number of side lobes decreases in apodized Bragg fiber gratings.

Another widely used apodization function is cosine one. The function is expressed by the formula [10]:

$$
g(z)=\cos ^{A}\left(\frac{\pi}{L_{g}} z\right)
$$

where $\mathrm{A}$ is the function parameter. Examples of profiles for different values of the parameter $A$ are given in the Figure 8.

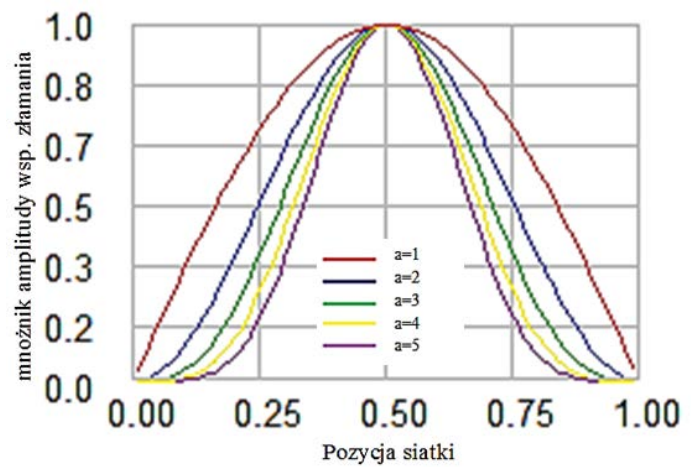

Fig. 10. Cosine apodization function for different values of the parameter A.

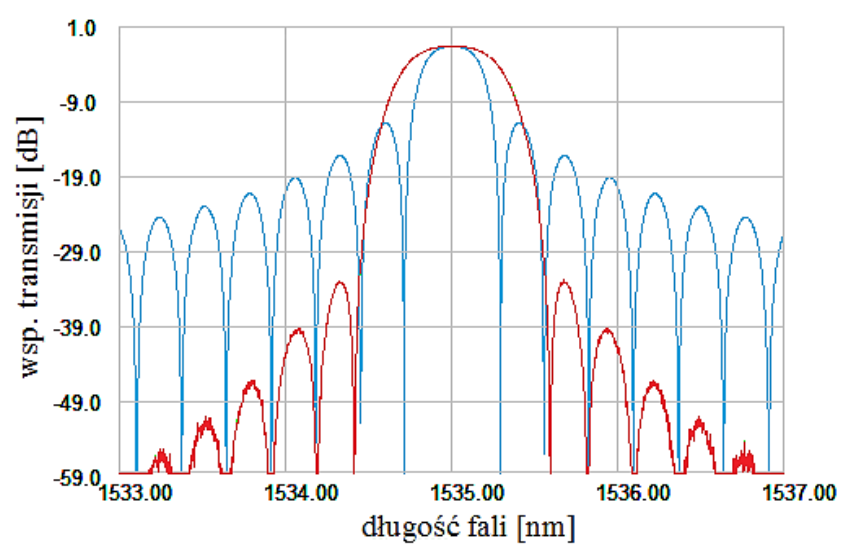

Fig. 11. Comparison of Bragg fiber gratings spectral characteristics without apodization - blue line and apodized cosine function for wsp. $\mathrm{A}=2$ - red line.

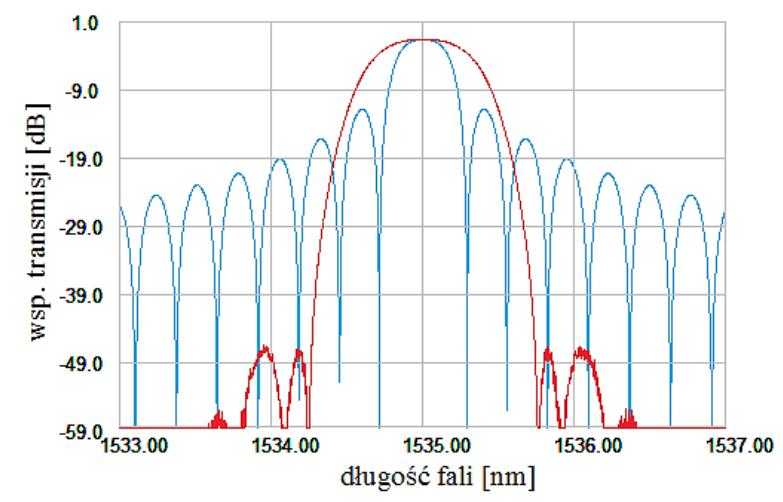

Fig. 12. Comparison of Bragg fiber gratings spectral characteristics without apodization - blue line and apodized cosine function for wsp. A = 3 - red line.

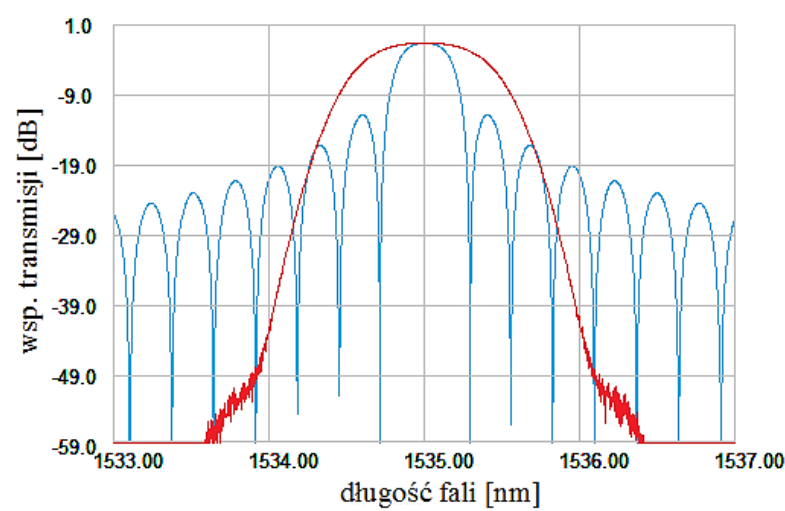

Fig. 13. Comparison of Bragg fiber gratings spectral characteristics without apodization - blue line and apodized cosine function for wsp. $\mathrm{A}=5$ - red line.

It is clearly seen from the charts that the side lobes are decreased in apodized gratings.

One more function is used for Bragg grating apodization, it is the so called Blackman function. The function is described by the formula [12]:

$$
g(z)=0,42+0,5 \cos \left(\frac{\pi z}{a}\right)+\frac{2}{25} \cos \left(\frac{2 \pi z}{a}\right)
$$


where $a$ is the function's parameter. Figure 14 shows Blackman function for different values of the parameter a.

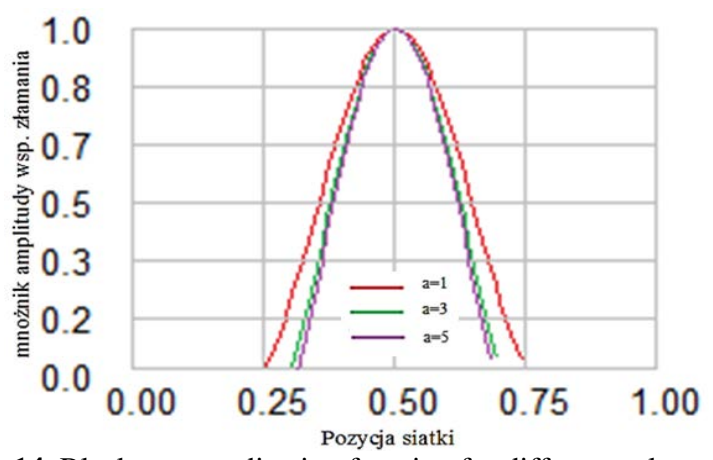

Fig. 14. Blackman apodization function for different values of the parameter $a$.

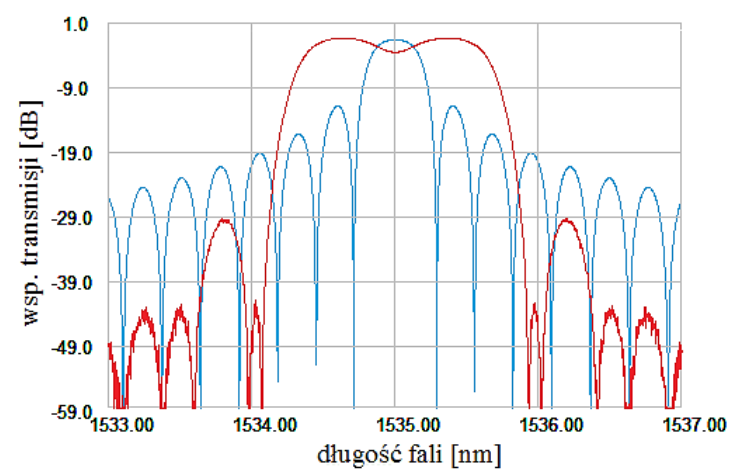

Fig. 15. Comparison of Bragg fiber gratings spectral characteristics without apodization - blue line and apodized Blackman function wsp. $a=2-$ red line.

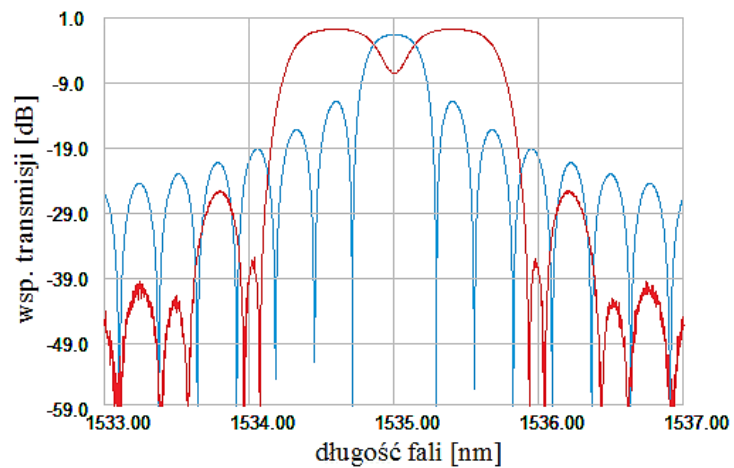

Fig. 16. Comparison of Bragg fiber gratings spectral characteristics without apodization - blue line and apodized Blackman function wsp. $a=5-$ red line.

In the Figure 14 you can see, that Blackman function does not bring to big changes comparing to Gauss function. An important factor in case of applying Blackman apodization function is occurrence of local minimum, which breaks down the base peak into two parts. Charts 15 and 16 show, that the value rise as well increases the importance of the minimum thereof.

One more is the sine function. This function differs from others by the fact that it has two parameters. The formula describes the function as follows [14]:

$$
g(z)=\operatorname{sinc}^{X}\left(\left|\frac{2(z-L / 2)}{L}\right|^{Y}\right)
$$

where $\mathrm{X}$ and $\mathrm{Y}$ are functional parameters.

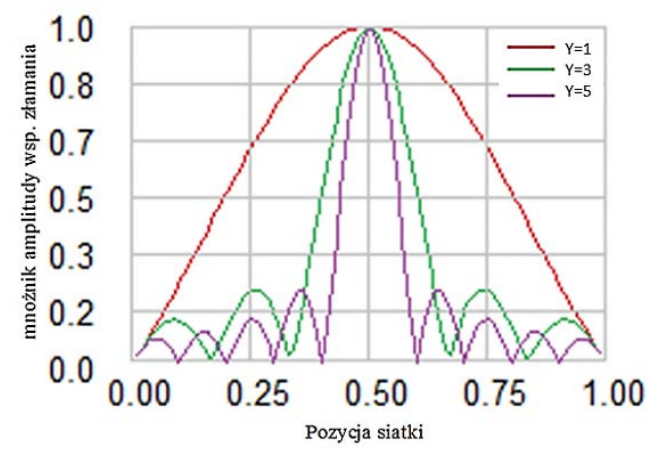

Fig. 17. Sine apodization function for different values of the parameter $\mathrm{Y}$ at constant value $\mathrm{X}=1$.

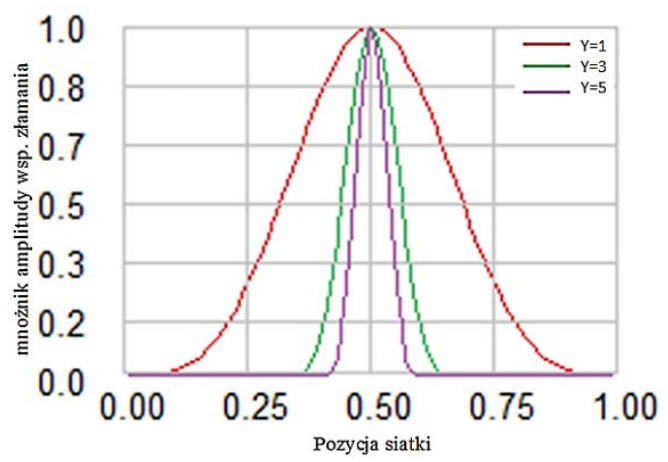

Fig. 18. Sine apodization function for different values of the parameter $\mathrm{Y}$ at constant value $\mathrm{X}=3$.

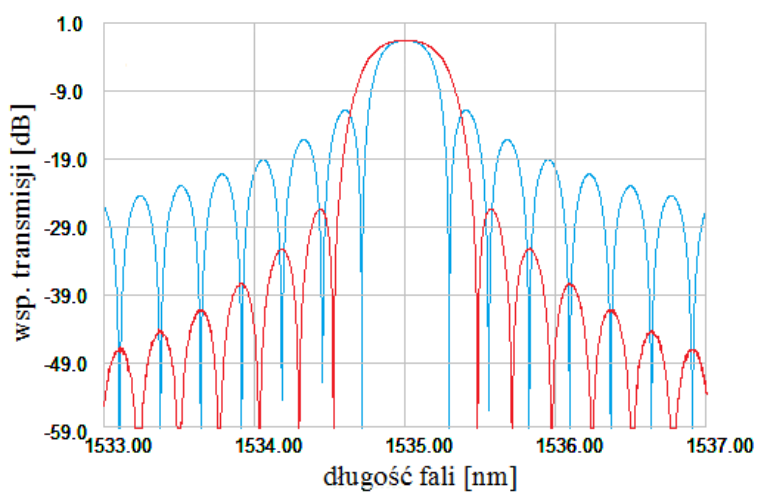

Fig. 19. Comparison of Bragg fiber gratings spectral characteristics without apodization - blue line and apodized sine function for wsp. $\mathrm{X}=1$ and $\mathrm{Y}=1$ - red line.

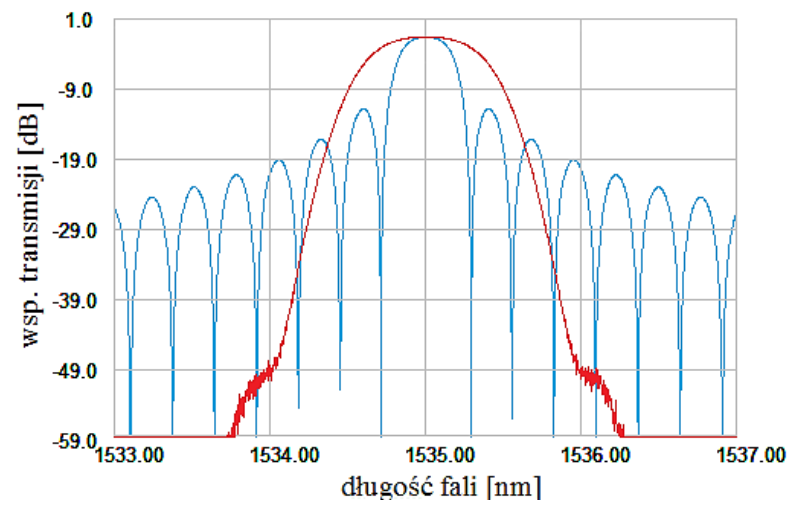

Fig. 20. Comparison of Bragg fiber gratings spectral characteristics without apodization - blue line and apodized Blackman function for wsp. $\mathrm{X}=3$ and $\mathrm{Y}=1$-red line. 


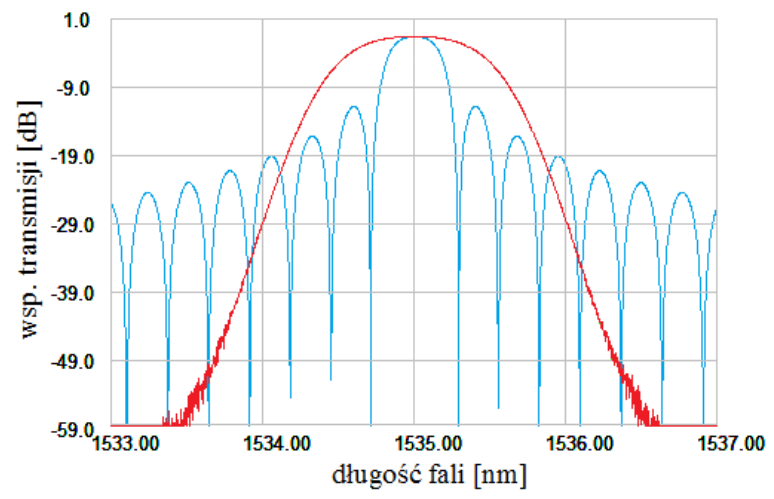

Fig. 21. Comparison of Bragg fiber gratings spectral characteristics without apodization - blue line and apodized sine function for wsp. $\mathrm{X}=5$ and $\mathrm{Y}=1-$ red line.

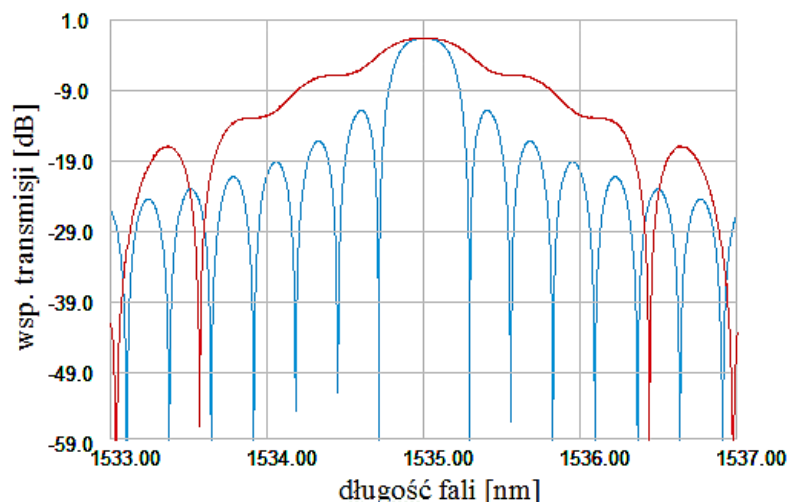

Fig. 22. Comparison of Bragg fiber gratings spectral characteristics without apodization - blue line and apodized sine function for wsp. $\mathrm{X}=1$ and $\mathrm{Y}=3$ - red line.

Figures 17 and 18 show, that in a proper way, in virtue of $\mathrm{X}$ and $\mathrm{Y}$ parameters change, it is possible to get several apodization profiles. At the same time from spectral characteristics charts in the figures $20-22$ it is seen, that the side lobes exclude the parameter increase, but they introduce noise characteristics. By contrast, with raising the parameter $\mathrm{Y}$ the side lobes become a part of the base peak, which sufficiently distorts the grating characteristics.

It is said above, that the important element defining the Bragg grating quality is the size of the so called side lobes occurring in its spectral characteristics. In order to define the grating quality let's introduce a new parameter which is called $\mathrm{S}_{\mathrm{GW}}$. This parameter is the ratio of the base peak power to the peak power of the biggest side lobe occurring in the grating characteristics. It is expressed by the following formula:

$$
S_{\mathrm{GW}}=\frac{P_{G}}{P_{W}}
$$

where $P_{G}$ is the base peak power; $P_{W}$ - peak power of the biggest side lobe. Definite Bragg grating characteristics with different Bragg waves and different values of reflection index and various grating lengths. The research assumes apodization of several most widely used functions and apodization functions factors change. Designed half-width and fiber strand width is 20 $\mathrm{dB}$. Figure 23 shows the means of $\mathrm{S}_{\mathrm{GW}}$ parameter to define capacity maximum peak of the first lobe density.
In contrast, Figure 24 shows the case when the higher order nets have the higher peak capacity.

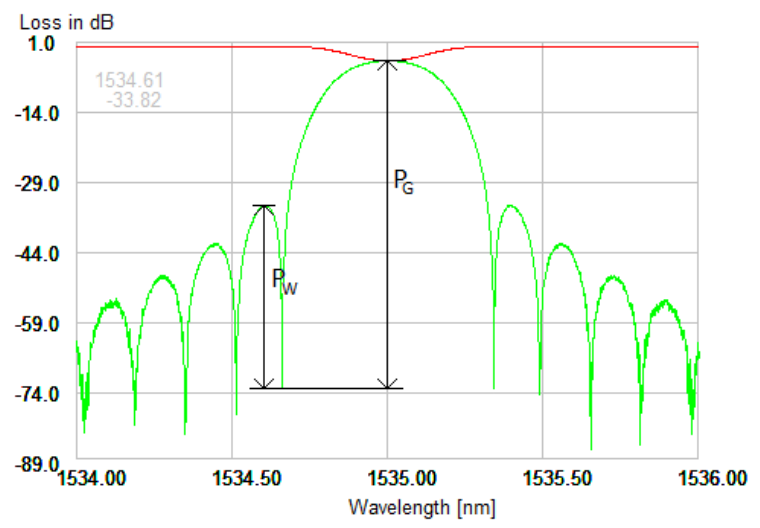

Fig. 23. Technique of defining $P_{G}$ and $P_{W}$, when the first-line peak capacity is high.

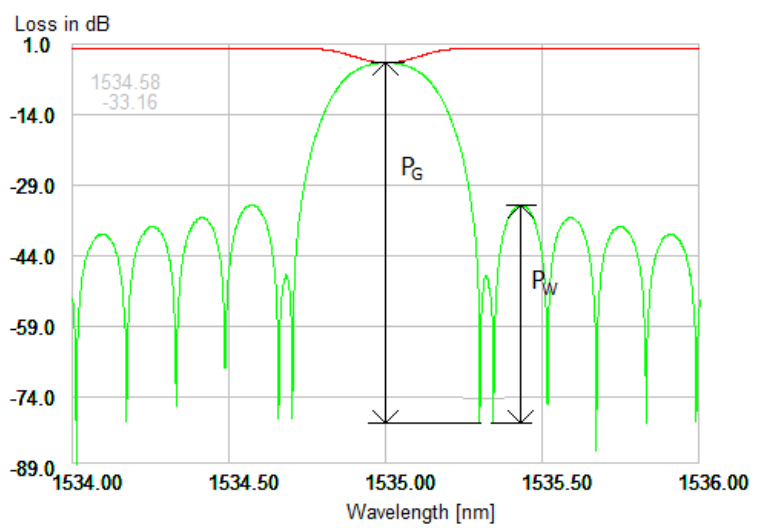

Fig. 24. Technique of defining $P_{G}$ and $P_{W}$, when the first-line peak capacity is less than the peak capacity of the higher order.

Side lobes analysis has been conducted using five base apodization functions In any case you will alter coefficient values of functions and data stored in the table. Modeling has been carried out for the fixed length value $\mathrm{L}=3 \mathrm{~mm}$ of the grating and reflection index $\mathrm{R}=69,8 \%$. Bragg wave length has been set at $1535 \mathrm{Nm}$. Each apodization function is provided with the table and chart of SGW index dependence on the apodization function. Due to the big size of the tables it was decided to show only functions charts in the work herein.

Figure 25 shows interrelation between $\mathrm{S}_{\mathrm{GW}}$ parameter and Gauss function parameter.

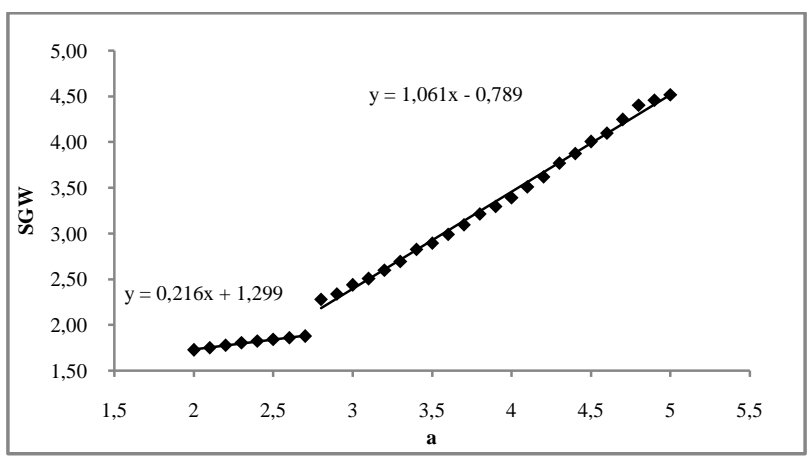

Fig. 25. Chart of the parameter $S_{\mathrm{GW}}$ and Gauss function parameter. 
Figure 25 shows that the chart is broken down into two parts. It is due to the fact, that the parameter $a=2,8$ of the first-line side lobes becomes a part of the base peak. The chart shows that the apodized grating peak value is the ultimate of the signal backward reflection. The linear function value is approximated with two functions. First curve:

$$
y=0,21 x+1,3
$$

Second curve:

$$
y=1,06 x-0,79 .
$$

Next function for research is the cosine function.

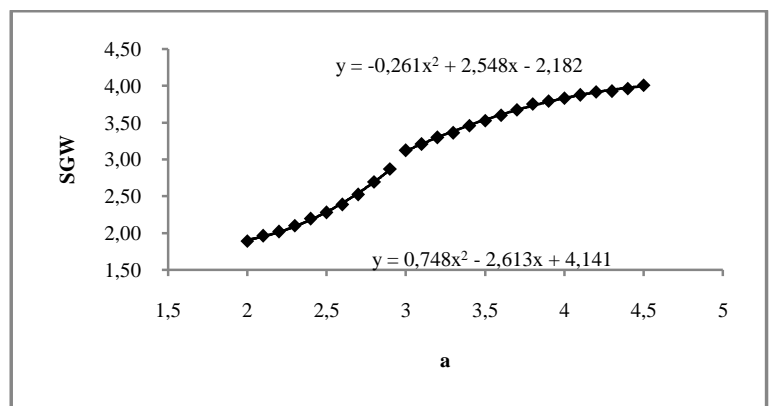

Fig. 26. Chart of $\mathrm{S}_{\mathrm{GW}}$ parameter and cosine function parameter.

Figure 26 shows that the scheme of dependences is broken down into two parts as in case of Gauss function. From the chart it is seen, that the first side lobes are decreased quicker, than the other succeedent. At value $a=3$, the side lobes of the second order become big.

In the chart it can also be seen, that at parameter increasing the side lobes capacity is decreased slower. At value $a=4,5$ the side lobes disappear completely. In spite of side lobes disappearance there exists rising noisiness, which distorts the spectrum characteristics. Dots on the chart are approximated by quadratic function.

First curve:

$$
y=0,75 x^{2}-2,61 x+4,14
$$

Second curve:

$$
y=-0,26 x^{2}+2,55 x-2,18
$$

Next part of the work shows modeling, conducted for apodized Bragg grating by means of Blackman function. Figure 27 shows the chart of the parameter $\mathrm{S}_{\mathrm{GW}}$ and Blackman function parameter.

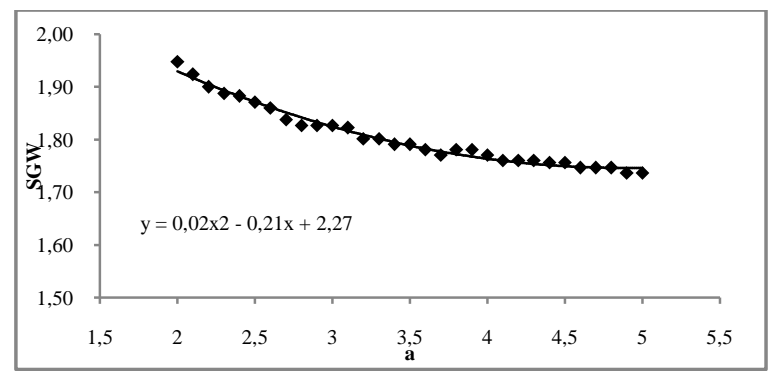

Fig. 26. Chart of $\mathrm{S}_{\mathrm{GW}}$ parameter and Blackman function parameter.
Figure 27 shows the parameter value, indicating decrease of $\mathrm{S}_{\mathrm{GW}}$ along with increase of parameter $a$.

In case of applying the Blackman apodization function the base peak consists of two parts with equal values of peak power. With increasing the function parameter the base peak is deepened. It gives the possibility to use Bragg apodized gratings with Blackman function for two different aims. Function value is approximated with the function, being described through the formula:

$$
y=x^{2}-0,21 x+2,27
$$

Another feature, subjected to modeling, in order to define the Bragg grating apodization quality is quadratic function. Figure 28 shows the chart of $\mathrm{S}_{\mathrm{GW}}$ parameter and quadratic function.

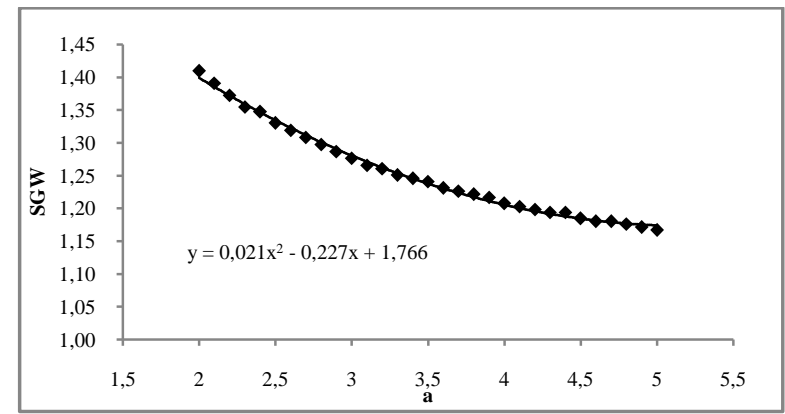

Fig. 28. Chart of $\mathrm{S}_{\mathrm{GW}}$ parameter $a$ and quadratic function.

Figure 28 shows that $\mathrm{S}_{\mathrm{GW}}$ parameter value sufficiently decreases with increase of the parameter $a$. That is, in order to eliminate the side lobes, the parameter 'a' shall be as small as possible comparing to a quadratic function. Apart from that, you can see that parameter $\mathrm{S}_{\mathrm{GW}}$ has the highest value, which equals to 1,41 . That is, quadratic function is not effective for eliminating the side lobes, as it is described further in the function section herein. Dots on the chart have been approximated with quadratic function according the preset formula:

$$
y=0,02 x^{2}-0,22 x+1,77
$$

As the final apodization function the sinc-function has been tested. This function has two parameters $\mathrm{X}$ and Y. Figure 29 shows the chart $3 \mathrm{D}$ of $\mathrm{S}_{\mathrm{GW}}$ parameter dependence on two functional parameters of sinc function.

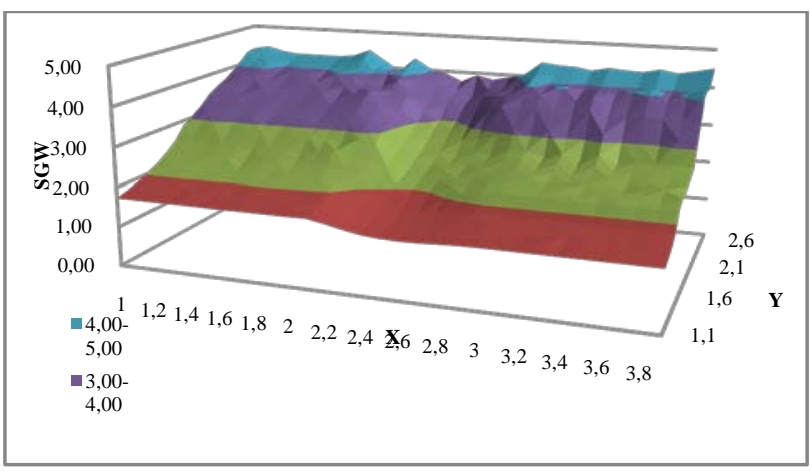


Fig. 29. Chart of the parameter $\mathrm{S}_{\mathrm{GW}}$ and parameters $\mathrm{X}$ and $\mathrm{Y}$ of the sinc- apodization function.

The chart on the Figure 29 shows, that the parameter change does not considerably affect the side lobes sizes. Exclusion is the local minimum for values of the parameter $X=2.4$. The chart shows, that with the parameter $\mathrm{Y}$ size growing the side lobes sufficiently decrease and the value of the parameter $\mathrm{Y}=2,8$ disappears completely leaving noise bands in the spectrum.

\section{Conclusion}

Proceeding from the above received outcomes of testing the different apodization functions with the aim of quality check and optimization of FBG spectral characteristics there can be drawn the following conclusions:

- the most proper functions for minimizing the side lobes are: Gauss function, cosine and sine functions;

- Bragg grating length does not influence at the side lobes size.

This work is supported by grant from the Ministry of Education and Science of the Republic of Kazakhstan within the framework of the Project № AP05132778 «Research and development of signals interrogation system with fiber-optic refractometer in telecommunication networks».

\section{References}

1. Kashyap R. Fiber Bragg gratings, Academic Press, (1999) London.

2. Vasiljyev S.A., Medvedkov O.I., Medvedkov I.G., Korolyev A.S., Bozhkov A.S., Kurkov Ye.M., Dianov (2005) Fiber gratings reflection index and their application, Quantum electronics, 35(12): 1085-1103.

3. Hill K.O., Fujii F., Johnson D.C., Kawasaki B.S. (1978) Photosensitivity on optical fiber waveguides; application to reflection filter fabrication, Applied Physycs Letter, 32: 647-649.

4. Kersey A.D., Davis M.A., Patrick H.J., LeBlanc M., Koo K.P., Askins C.G., Putnam M.A., Friebele E.J. (1997) Fiber grating sensors, Light wave Technology, 15(8): 1442-1463.

5. Lopez-Higuera J.M. (2002) Handbook of optical fiber sensing technology, John Wiley \& sons, P. 795.

6. Bilodeau F., Johnson D.C., Theriault S., Malo B., Albert J., Hill K.O. (1995) An all-fiber densewavelength multiplexer/DE multiplexer using photo imprinted Bragg gratings, IEEE Photonics Technology Letters, 7(4):388-390,.

7. Chai J., Yu Z., Liu Y. (2006) Analysis of the apodization parameter of linearly chirped Bragg gratings for dispersion compensation, School of Science, Beijing University of Posts and Telecommunications, Beijing.
8. Zhang H. (2014) A novel method of optimal apodization selection for chirped fiber Bragg gratings, Optik 125, 1646- 1649.

9. Khan S., Islam S. (2012) Determination of the Best Apodization Function and Grating Length of Linearly Chirped Fiber Bragg Grating for Dispersion Compensation, Bangladesh University of Engineering and Technology, Bangladesz.

10. Wójcik W., Kashaganova G.B. (2015) Study of Bragg fiber gratings spectral characteristics, Materials of the II International scientific-practical conference «Information and telecommunication technologies: education, science, practice», Almaty, Kazakhstan, II vol., pp. 77-81.

11. Kashaganova G.B. (2016) The influence of various apodization functions on the spectral characteristics of fiber Bragg gratings, Bulletin of KazNTU named after Satbayev K.I., 3(115):302-311

12. Li X., Chen X., Yin Y. and Xie S. (2003) A novel apodization technique of variable mark-space ratio for fabricating sampled fiber bragg grating, Tsinghua University, China.

13. Albert J. et al. (1995) Apodisation of the spectral response of fibre Bragg grat-ings using a phase mask with variable diffraction efficiency, Electronic Letters, 31: 222-223.

14. Malo B., et al. (1995) Apodised in-fiber Bragg grating reflectors photoim- printed using a phase mask, Electronic Letters, 31: 223-225.

15. Pastor D. et al. (1996) Design of apodized linearly chirped fiber gratings for dispersion compensation, J. Lightwave Technology, 14: 2581-2588.

16. Keitmann-Curdes O., Brendel B., Marg C., Ermert H. (2002) Optimization of apodization based on the sidelobe pressure energy in simulated ultrasound fields, in: Proc. IEEE Int. Ultrason. Symp.,16771680.

17. Abdullina S.R., Babin S.A., Kablukov S.I., Vlasov A.A. (2007) Simple technique of fiber Bragg gratings apodization by use Proc, Laser Optics 2006: Diode Lasers and Telecommunication Systems, ed. Nikolay N. Rosanov, 6612: 661201 (1-10).

18. Chuang K.P. et al. (2004) Pure apodized phaseshifted fiber Bragg gratings fabricated by a two-beam interferometer with polarization control, IEEE Photonics Technology Letters, 16: 834-836.

19. Othonos A., Kalli K. (1999) Fiber Bragg gratings. Artech House.

20. Albert J., Hill K. O., Malo B. (1995) Apodization of spectral response of fiber Bragg gratings using phase mask with variable diffraction efficiency, Electronic Letters, 31(3): 222-223. 